\section{An unusual long-delay echo}

SIR-Long-delay echoes (LDEs) are weak signals heard at times ranging from several hundred milliseconds to several seconds after an initial radio transmission. They were first reported in the late 1920 s (refs 1-3) and have often been reported since (see ref. 4). But they are elusive, and might be considered as an example of "pathological science"

I carried out a search for LDEs on the 28- $\mathrm{MHz}$ amateur band each evening from 18 to 27 December 1989 . On the evening of 20 December at 20:05 eastern standard time I heard and recorded an extraordinary example of a sextuple LDE. The multiple echoes, resulting from a singlesideband (SSB) transmission, were weak but distinct.

The transmitted signal (about $1 \mathrm{~kW}$ into a 5-element Yagi-Uda antenna oriented west-northwest) was the word "tango". Weak but distinct reproductions of my voice can be heard at $2.9,4.5,5.5,7.1,8.1$ and $9.5 \mathrm{~s}$. I timed the echoes by repeatedly playing back the tape into a storage oscilloscope and manually marking the trace of a particular echo. I estimate the uncertainties in these times to be of the order of $0.1 \mathrm{~s}$. The first and fourth echoes are harder
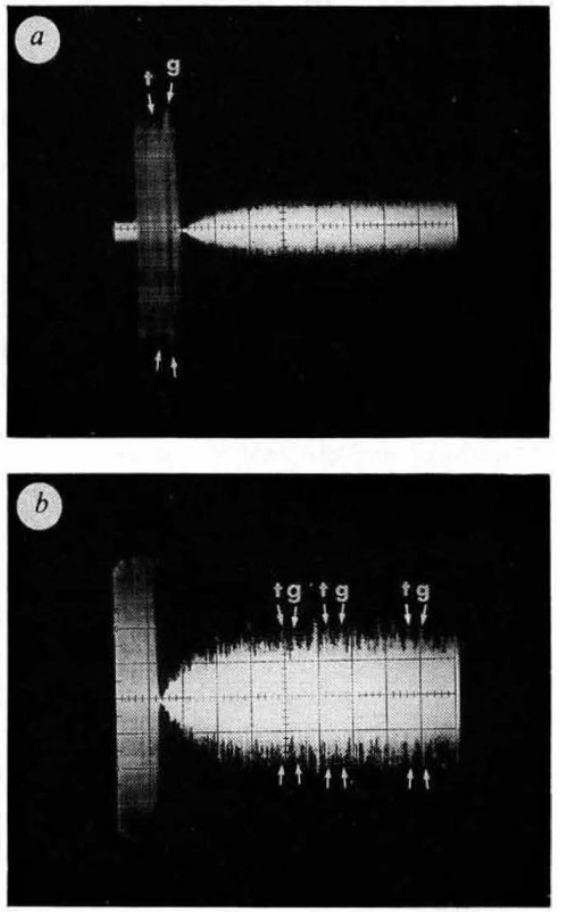

a, Unfiltered receiver output consisting of transmitted single-sideband signal "tango" and noise. Syllables "tan" and "go" are marked by the arrows $t$ and $g$, respectively. $b$, Filtered receiver output. Times at which the syllables "tan" and "go" can be heard on the tape recording are marked approximately by the arrows $t$ and $g$, respectively. See text for details. Duration of the oscilloscope trace is $10 \mathrm{~s}$ in each photograph. Copies of the magnetic tape cassette recording available on request from A.K.G. to hear than the others: the second seems to be Doppler-shifted up very slightly in frequency. The receiver output ( $a$ in the figure) has a bandwidth of about $2.5 \mathrm{kHz}$ and so the echoes are concealed in the noise. To enhance the echoes, I played the tape-recordings through a narrow-band audio filter, about $150-\mathrm{Hz}$ wide, at about $900 \mathrm{~Hz}$ ( $b$ in the figure). This presentation is not well suited for displaying these weak echoes but, in the case of the stronger echoes, the syllables "tan" and "go" can be heard at the times indicated.

I am certain that this example of an LDE is not an artefact. I had previously used the magnetic tape cassette to record Morse code but not SSB signals, so the echoes are not signals previously recorded and incompletely erased. The transfer of a strong audio signal to an adjacent layer of magnetic tape is a recognized problem but a weak overprint is, because of the geometry of the tape cassette, never subsequently heard on the tape more than $1.5-3 \mathrm{~s}$ before or after the strong transmitted signal. I cannot exclude the possibility that the echo at $2.9 \mathrm{~s}$ is an overprint, but no echoes are heard on the tape after the next two transmitted words "lima" and "echo".

Any LDE may be a hoax by which a radio operator transmits a signal, but in this case I had initially adjusted my transmitter at high power at one frequency and then changed to a new operating frequency several kilohertz away. The word "tango" was my first SSB transmission of the evening and it is extremely unlikely that anyone would have so quickly found my new frequency, recorded my signal and re-transmitted it six times over.

The intervals between successive echoes are $1.6,1.0,1.6,1.0$ and $1.4 \mathrm{~s}$. Because the echoes are too weak for me to time accurately, I cannot vouch for a perfect regularity in the delay times, but my previous observations of LDEs suggest that some sort of periodic structure exists in LDE delay times ${ }^{6.7}$

I believe that LDEs are relatively common, and that a systematic search at high power may provide sufficient data to discover how radio waves can persist essentially undistorted for such long periods of time.

\section{Amateur Radio Station VE3HX}

A. K. GOODACRE 1286 Woodside Drive,

Ottawa, Ontario K2C 2G9,

Canada

1. Stormer, C. Nature 122, 681 (1928)

2. Stormer, C. Proc. R. Soc. Edin. 50, 187-199 (1930).

3. Galle, J.-B. \& Talon, G. C. r. hebd. Séanc. Acad. Sci., Paris $190,48-50$ (1930)

4. Muldrew, D.B. J. geophys. Res. 84, 5199-5215 (1979)

5. Langmuir, I. Phys. Today 42, 36-48 (1989).

6. Goodacre, A.K. J. geophys. Res. 85, 2329-2334 (1980) Goodacre, A.K. QST 64, 14-16 (1980)

\section{Neurotransmitter identity doubt}

SIR-In a recent Letter $^{1}$, Bult et al. showed that stimulation by an electric field of the isolated and perfused canine ileocolonic junction pretreated with adrenergic and cholinergic blocking agents results in the release of nitric oxide. The authors concluded that nitric oxide is released from the nonadrenergicnoncholinergic neurons innervating this tissue and, therefore, that nitric oxide is the inhibitory transmitter for these neurons. An alternative explanation entirely consistent with these data is that stimulation of these neurons causes release of an unknown neurotransmitter that, in turn, stimulates the underlying smooth muscle to generate nitric oxide.

We find that stimulation of isolated strips of rabbit corpus cavernosum pretreated with guanethidine and atropine promotes the endogenous formation and release of NO, nitrite and cyclic GMP. Formation of these substances in tissues is frequency-dependent, associated with frequency-dependent relaxation of corporal smooth muscle, and all responses are abolished by tetrodotoxin and unaffected by indomethacin. We found that stimulation-induced relaxation of corporal smooth muscle is markedly inhibited by $N^{\mathrm{G}}$-nitro-L-arginine, an inhibitor of the biosynthesis of NO from L-arginine ${ }^{2}$, and by haemoglobin and methylene blue, agents that are known to interfere with the biological actions of NO (refs 3 , 4 ; see figure).

The inhibitory effects of $N^{\mathrm{G}}$-nitro-Larginine, $N^{\mathrm{G}}$-amino-L-arginine and $N^{\mathrm{G}}$ methyl-L-arginine were nearly completely reversed by addition of excess L-arginine but not $\mathrm{D}$-arginine. These observations suggest that these $N^{\mathrm{G}}$-substituted analogues of L-arginine inhibit formation of NO from endogenous L-arginine. Stimulation-induced corporal smoothmuscle relaxation is accompanied by sixto eight-fold increases in tissue levels of nitrite, the spontaneous oxidation product of NO (ref. 5) and cyclic GMP, the intracellular mediator of smooth muscle relaxation caused by NO (ref. 6). Both of these responses are nearly abolished by the application of $10 \mu \mathrm{M} N^{\mathrm{G}}$-nitroL-arginine.

Because penile erection is the result of neurogenetically stimulated relaxation of corpus cavernosum smooth muscle follwed by venous occlusion and engorgement of corporal sinusoids with blood, these observations, suggest that penile erection is mediated by NO generated in response to nonadrenergic-adrenergic neurotransmission. Although we have found that stimulation-induced corporal smooth muscle relaxation occurs independently of vascular endothelium, 